\title{
Lipid Synthesis Is a Metabolic Liability of Non-Small Cell Lung Cancer
}

\author{
Robert U. Svensson and Reuben J. Shaw \\ Molecular and Cell Biology Laboratory, The Salk Institute for Biological Studies, \\ La Jolla, California 92037 \\ Correspondence: shaw@salk.edu
}

\begin{abstract}
The renaissance in the study of cancer metabolism has refocused efforts to identify and target metabolic dependencies of tumors as an approach for cancer therapy. One of the unique metabolic requirements that cancer cells possess to sustain their biosynthetic growth demands is altered fatty acid metabolism, in particular the synthesis of de novo fatty acids that are required as cellular building blocks to support cell division. Enhanced fatty acid synthesis that is observed in many tumor types has been postulated to open a therapeutic window for cancer therapy and, correspondingly, efforts to pharmacologically inhibit key enzymes of fatty acid synthesis are being pursued. However, despite these efforts, whether inhibition of fatty acid synthesis stunts tumor growth in vivo has been poorly understood. In this review, we focus on the recent evidence that pharmacologic inhibition of acetyl-CoA carboxylase, the enzyme that regulates the rate-limiting step of de novo fatty acid synthesis, exposes a metabolic liability of non-small cell lung cancer and represses tumor growth in preclinical models.
\end{abstract}

\section{ALTERED FATTY ACID SYNTHESIS IN CANCER}

Although most normal cells and tissues satisfy their requirement for fatty acids by importing them from the circulation, the observation more than 60 years ago that tumors undergo high rates of de novo lipogenesis suggested that cancer cells satisfy their lipid demand differently compared with their normal counterparts (Medes et al. 1953). Surprisingly, the rate of lipogenesis measured in tumors was comparable to that of the liver, an organ that requires a high rate of fatty acid (FA) synthesis. Since then, enhanced FA synthesis has been documented in a number of tumors (reviewed in Swinnen et al. 2006; Abramson 2011). The elevated de novo FA synthesis observed in tumors is reflected by the increased expression of lipogenic enzymes that has been described in a number of cancers (Szutowicz et al. 1979; Witters et al. 1994; Milgraum et al. 1997). A seminal finding in this respect was the identification of the oncoprotein OA-519 as fatty acid synthase (FASN), which was overexpressed in aggressive breast cancer (Kuhajda et al. 1994) and now confirmed to be up-regulated in a wide number of other cancers such as those of the prostate, ovary, colon, lung, and bladder (Menendez and Lupu 2007).

Lipogenic enzymes in the FA synthesis pathway are regulated at various levels. Strikingly, many of the genes encoding these enzymes are controlled transcriptionally by the sterol regulatory element-binding proteins (SREBPs) (Fig. 1). SREBPs are basic helix-loop-helix transcription factors that are crucial for maintaining cellular lipid homeostasis (Horton 2002). There are three SREBP isoforms in mammals: SREBP1a and SREBP1c, which are products of the $S R E B F 1$ gene, and SREBP2, a product of the SREBPF2 gene. Although SREBP1c mainly regulates genes in the FA synthesis pathway and SREBP2 regulates genes involved in cholesterol synthesis, redundancy among the SREBPs allows each isoform to regulate genes within each pathway (Horton 2002). Inhibition of SREBP1 and SREBP2 in cancer cells led to altered cellular lipid composition, which triggered endoplasmic reticulum (ER) stress and the unfolded protein response (UPR) (Griffiths et al. 2013), demonstrating that SREBPs play a critical role in maintaining cellular lipid homeostasis in cancer. Emerging evidence also indicates that SREBPs provide a crucial link between oncogene signaling and altered fatty acid metabolism. SREBP1 activity is induced by AKT in a manner that is dependent on mTORC1 (mammalian target of rapamycin complex 1), demonstrating that lipogenesis and cell growth are intimately connected through the AKT-mTORC1 pathway (Fig. 1; Porstmann et al. 2008; Duvel et al. 2010).

In addition to transcriptional regulation, the activity of lipogenic enzymes is modulated posttranslationally by phosphorylation. For example, AKT directly phosphorylates and stimulates ATP citrate lyase (ACLY) (Bauer et al. 2005) contributing to the control of histone acetylation in response to growth factor stimulation (Lee et al. 2014). In contrast to this phosphorylation stimulating ACLY by oncogenic signaling, in response to low energy levels, the AMP activated protein kinase (AMPK) phosphorylates and inactivates key regulators of FA synthesis, including acetyl-CoA carboxylase 1 and 2 (ACC1 and ACC2) as well as SREBP1c itself (Fig. 1; Carling et al. 1989; Li et al. 2011). Thus, during periods of energy deprivation, AMPK dominantly inhibits lipogenesis. The acti-

(C) 2016 Svensson and Shaw. This article is distributed under the terms of the Creative Commons Attribution-NonCommercial License, which permits reuse and redistribution, except for commercial purposes, provided that the original author and source are credited. 


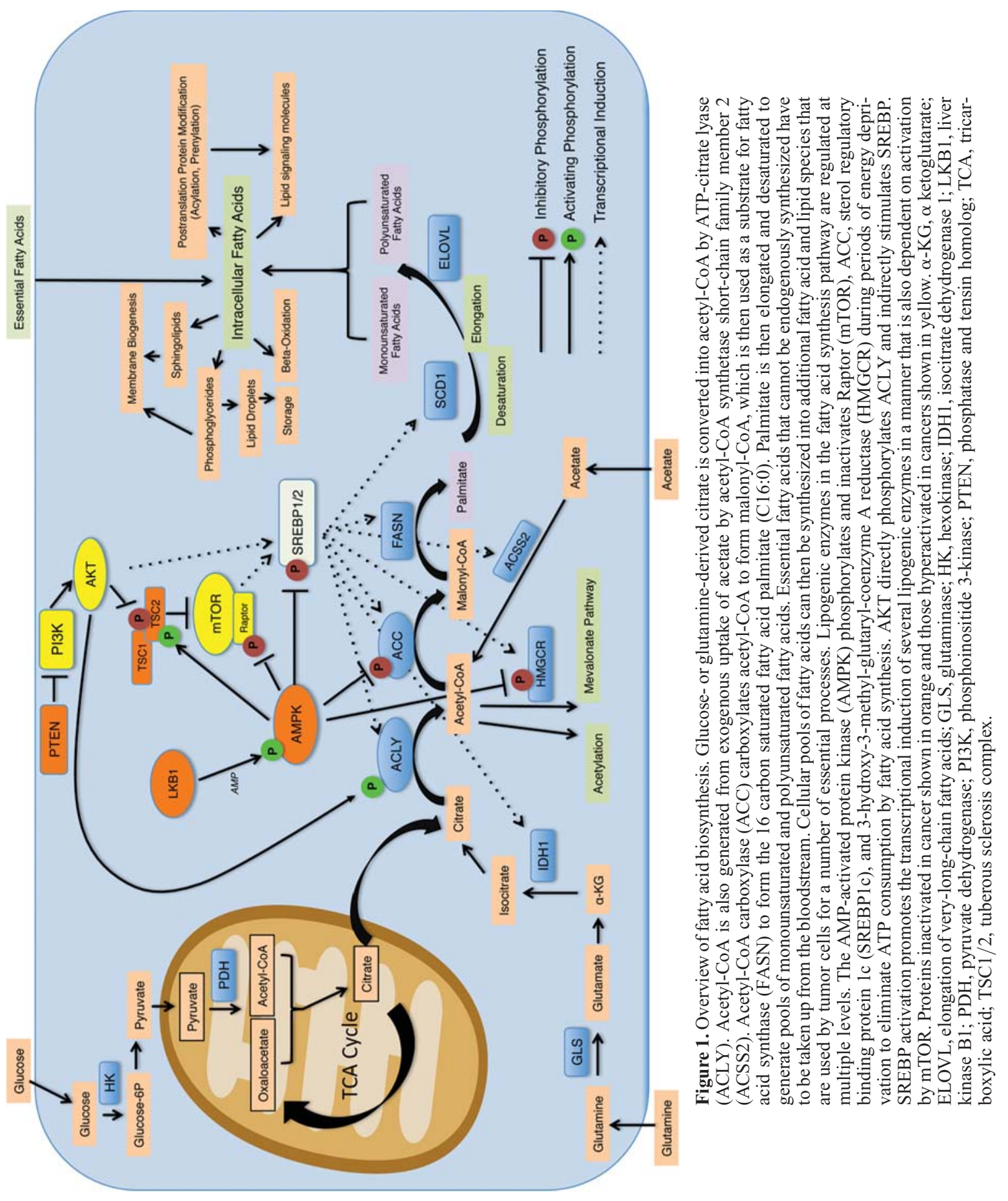


vation of AMPK is induced by phosphorylation by the tumor suppressor serine threonine kinase 11 (STK11), otherwise known as liver kinase B1 (LKB1), suggesting that ACC activity and lipogenesis itself may be hyperactivated in the broad variety of cancers lacking this tumor suppressor (Shackelford and Shaw 2009), paralleling the hyperactivation of ACLY predicted in the many cancers mutated for AKT, PIK3CA (phosphatidylinositol-4,5biphosphate 3-kinase catalytic subunit alpha), or PTEN (phosphatase and tensin homolog).

Generally, the importance of FA synthesis in cancer cell proliferation and survival in vitro has now been described in a number of studies (Rohrig and Schulze 2016). However, whether autochthonous tumors in vivo possess a similar requirement is less understood. Because of the complex nature of tumors and the availability of lipids from both the circulation and the tumor microenvironment, the effects of de novo FA synthesis inhibition in vitro may not be translatable in vivo. Furthermore, the need to rigorously evaluate the anticancer effects of FA synthesis inhibitors is required to understand whether the enhanced synthesis observed in tumors is truly a metabolic dependency of cancer or rather simply a byproduct of altered oncogene signaling and metabolism (Kim and DeBerardinis 2016).

\section{FASN: THE FIRST TARGETED COMPONENT OF THE FA SYNTHESIS PATHWAY}

Given elevated FASN was the first molecular discovery linking fat metabolism to cancer (Kuhajda et al. 1994), many studies have since shown increased FASN expression and enhanced activity in a wide variety of tumors (Menendez and Lupu 2007). Correspondingly, efforts focused on pharmacologically inhibiting FASN activity have been pursued for a number of years, and several FASN inhibitors have been described to date. The mycotoxin cerulenin inhibits the activity of FASN and has shown the ability to inhibit proliferation and induce apoptosis of cancer cells and xenograft tumors in vivo (Pizer et al. 1996; Menendez et al. 2004). Similarly, the more stable analog, C75, displayed antitumor activity in xenograft models of breast, prostate, and lung cancer (Pizer et al. 2000; Chen et al. 2012; Relat et al. 2012). C75 was also shown to significantly delay breast cancer progression in HER2 transgenic mice (Alli et al. 2005). TVB3166 , a potent and orally bioavailable inhibitor of FASN, induced apoptosis in breast and prostate cancer cell lines and displayed antitumor activity in several tumor xenograft models (Ventura et al. 2015). The thiophenopyrimidine-based FASN inhibitor Fasnall recently showed potent antitumor activity in the mouse mammary tumor virus (MMTV)-Neu model of HER2 ${ }^{+}$breast cancer, where it also synergized with carboplatin (Alwarawrah et al. 2016). Collectively, the preclinical activity of FASN inhibitors has been encouraging, and recently the first FASN inhibitor was entered into clinical trials for cancer. However, the mechanism underlying the antitumor efficacy of various FASN inhibitors is poorly understood, as FASN inhibition was shown in some settings to increase malonyl-CoA levels (Thupari et al. 2001), which led to reduced food intake and body weight in mice (Loftus et al. 2000). Newer studies have also shed light on multiple mechanisms underlying sensitivity to FASN inhibitors (Benjamin et al. 2015; Ventura et al. 2015).

\section{ACLY AND ACSS2: MORE THAN ONE WAY TO MAKE ACETYL-CoA}

Other enzymes within the FA synthesis pathway have been hypothesized to be attractive therapeutic targets in cancer. In the cytoplasm, glucose- or glutamine-derived citrate is converted into acetyl-CoA by ACLY (Fig. 1). Acetyl-CoA is an essential substrate for FA synthesis; hence, ACLY inhibition should result in depleted acetylCoA pools and offer a way to shut down FA synthesis in cancer. Inhibition of ACLY by either RNAi or pharmacological inhibitors results in cell cycle arrest and induction of apoptosis in vitro and in vivo (Hatzivassiliou et al. 2005). However, whether these effects are specifically due to loss of FA synthesis in cancer cells is questioned, as fatty acid supplementation did not rescue the effects of ACLY inhibition (Migita et al. 2008). Moreover, given that acetyl-CoA contributes to the mevalonate pathway as well as to histone acetylation (Wellen et al. 2009), ACLY activity may modulate cancer growth on multiple levels.

Importantly, ACLY activity does not provide the only source of cellular acetyl-CoA. The enzyme acetyl-CoA synthetase short-chain family member 2 (ACSS2) produces acetyl-CoA using acetate as a substrate (Fig. 1; Yoshii et al. 2009; Comerford et al. 2014). Thus, ACSS2 may compensate for ACLY inhibition if cellular pools of acetate are not limiting. To this end, exogenous treatment of cancer cells with acetate was able to rescue the effects of ACLY inhibition on proliferation arrest and histone acetylation (Wellen et al. 2009; Hanai et al. 2012). Furthermore, ACSS2 expression is up-regulated in ACLYdeficient cells in vitro and in vivo, suggesting that acetate metabolism underlies a crucial mechanism for ACLY deficiency (Zhao et al. 2016). The contribution of acetate to acetyl-CoA production plays a fundamental role in intermediary metabolism and proliferation of cancer cells (Lyssiotis and Cantley 2014). Exogenous acetate is readily captured and metabolized by cancer cells to fuel cell growth and interestingly was found to contribute a greater proportion of carbon to the acetyl-CoA pool than glucose or glutamine (Mashimo et al. 2014). ACSS2 has been shown to be essential in this process, as ACSS2 deletion in mouse models of hepatocellular carcinoma led to a significant reduction in tumor burden (Comerford et al. 2014). Additionally, ACSS2 contributes to acetyl-CoA generation during hypoxia and is essential for cancer cell survival in tumor xenografts (Schug et al. 2015). Taken together, the pivotal roles of ACLY and ACSS2 in generating acetyl-CoA pools that are required for cancer cell growth make them attractive therapeutic targets for cancer. However, because of their relative redundancies in maintaining these pools and the ability of cancer cells to readily uptake exogenous sources of acetate in vivo, whether pharmacological inhibition of either ACLY or 
ACSS2 will provide a maximal therapeutic benefit still needs to be fully analyzed.

\section{ACC: A CANCER TARGET WITH A DISTINCT ADVANTAGE?}

An alternative approach to inhibit FA synthesis in cancer is the pharmacological inhibition of ACC, which controls the enzymatic step in between ACLY and FASN; the first-committed step of the lipogenesis pathway. ACC inhibition may offer a favorable advantage over other lipogenic enzymes because of its critical role in controlling the rate-limiting reaction of FA synthesis, the production of malonyl-CoA, which serves as the substrate for the de novo synthesis of all endogenously made fatty acids (Fig. 1). ACC uses acetyl-CoA for malonyl-CoA production regardless of the source from which it was derived. Thus the generation of malonyl-CoA pools that are essential for FA production depends entirely on the activity of ACC and is not confounded by functional redundancies among other lipogenic enzymes such as ACLY and ACSS2. Furthermore, inhibition of ACCs should lead to greater lipid depletion than inhibition of any other lipogenic enzyme in the pathway, because of loss of FA synthesis and simultaneous induction of fatty acid oxidation (FAOxn) that occurs when ACC is inhibited (see below). Correspondingly, efforts to target ACC have been pursued in the bio-pharmaceutical industry for the past two decades; however, until recently the development of potent and specific ACC inhibitors with favorable drug-like properties has been difficult (reviewed in Tong and Harwood 2006; Abramson 2011). Here we focus on the recent development of a novel small-molecule allosteric ACC inhibitor that has shown promise as an anticancer compound in preclinical models of non-small cell lung cancer (NSCLC) (Svensson et al. 2016).

\section{ACC REGULATION OF FATTY ACID METABOLISM}

Eukaryotic ACCs are large, single-chain multidomain enzymes with a biotin carboxylase (BC) domain, a biotin carboxyl carrier protein (BCCP) domain, and a carboxyltransferase (CT) domain (Fig. 1). ACC catalyzes the ATP-dependent carboxylation of acetyl-CoA to form malonyl-CoA, which is the first committed step in fatty acid synthesis and the rate-limiting reaction in the pathway (Kim 1997). The carboxylation of acetyl-CoA to malonyl-CoA is catalyzed by the BC domain of ACC and involves the ATP-dependent carboxylation of biotin with bicarbonate serving as the donor of $\mathrm{CO}_{2}$. CT then transfers the carboxyl group from biotin to acetyl-CoA to form malonyl-CoA. In mammals, ACC exists as two isoforms, a cytoplasmic $265-\mathrm{kDa}$ ACC1 isoform that is expressed in lipogenic tissues such as the liver and a 280-kDa ACC2 isoform expressed in oxidative tissues such as heart and muscle, where it is localized exclusively to the outer mitochondrial membrane (Bianchi et al. 1990; Kim 1997).
Dimerization of ACC is required for enzymatic activation, and the active form of mammalian ACC is a large linear polymer. Elegant studies have shown that ACC forms multiple dimers within the $\mathrm{BC}$ domain of the enzyme, which promotes its activity as a carboxylase to enable the generation of malonyl-CoA (Fig. 1; Shen et al. 2004; Cho et al. 2008). The original model in the field was that the malonyl-CoA produced from ACC1 serves as a substrate for FASN and results in the formation of fatty acids, and the malonyl-CoA produced by ACC2 at the outer mitochondrial membrane functions to allosterically inhibit carnitine palmitoyltransferase 1 (CPT-1) to prevent fatty acid entry into mitochondria to undergo $\beta$-oxidation (McGarry et al. 1978). However, recent findings from genetic knockout and knock-in mice with point mutations resulting in mild gain-of-function alleles of ACC reveal that this strict division of ACC isoforms in regulation of FA synthesis and FAOxn is probably not accurate, as the evidence suggests that there are substantial redundancies between each ACC isoform (Savage et al. 2006; Harada et al. 2007; Fullerton et al. 2013). Thus, the ability of malonyl-CoA to promote fatty acid synthesis or to inhibit FAOxn independent of the ACC isoform may be an important feature for tumor cell adaptation. However, regardless of the acetyl-CoA and malonyl-CoA source, the net effect of ACC activation in a tumor results in enhanced fatty acid synthesis and reduced fatty acid degradation via FAOxn inhibition (Fig. 2A). The de novo synthesis of intracellular fatty acids results in the production of fatty acids and lipids that can be used by tumor cells for a number of purposesincluding membrane biogenesis, posttranslational modification of oncoproteins, to function as signaling molecules, or stored for energy production - all of which favor the growth of a tumor (Fig. 1).

Physiological inhibition of ACC activity is controlled by phosphorylation by AMPK (Steinberg and Kemp 2009). AMPK is activated under conditions of low ATP, such as following nutrient deprivation or mitochondrial inhibitors, but also modulated by metabolic hormones and whole-body effects from exercise and dietary restriction. AMPK phosphorylates conserved serine residues Ser79 in mouse ACC1/Ser212 in ACC2 to inhibit its dimerization that is required for activation. In addition, ACC is activated allosterically by citrate, which provides the signal that acetyl-CoA pools are sufficient to enable FA synthesis. Citrate promotes dimerization of ACC and dephosphorylation of the AMPK phosphorylated serines, which serves to induce ACC activity and malonyl-CoA formation. The stimulatory effect of citrate on ACC is reversed by palmitoyl-CoA, which is abundant when there is an excess of fatty acids and therefore functions to promote the inactive conformation of ACC.

\section{ACC AS AN ANTICANCER DRUG TARGET}

Several lines of evidence support the rational for targeted ACC inhibition in oncology. Short interfering RNA (siRNA) knockdown of ACC1 expression induced apo- 


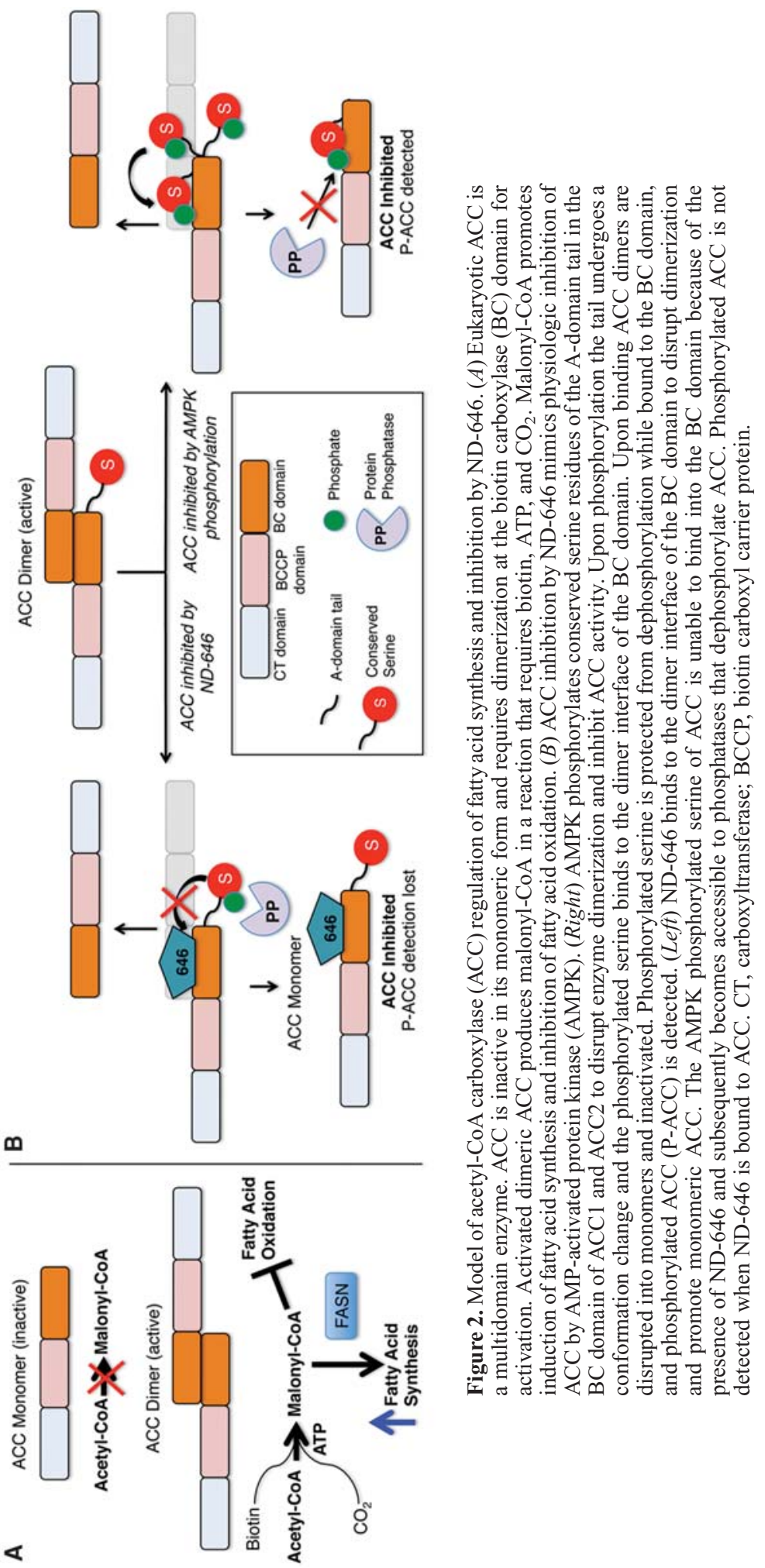


ptosis in prostate (Brusselmans et al. 2005) and breast (Chajes et al. 2006) cancer cells and in cisplatin-resistant lung cancer cell lines (Wangpaichitr et al. 2012), and chemical inhibition of ACC1 and ACC2 by the macrocyclic myxobacterial natural product Soraphen A led to growth arrest in breast cancer cells (Beckers et al. 2007) and induction of apoptosis in prostate cancer cells (Corominas-Faja et al. 2014). Constitutively active ACC mutants also protected head and neck squamous cancer cells from cetuximab-induced growth inhibition (Luo et al. 2016). However, although these observations provide compelling data that ACC mediates cellular proliferation, these studies were exclusively in vitro and did not address whether ACC activation was required for tumor growth in vivo, as the genetic role of ACC in tumor growth had not been investigated. In an attempt to understand this and also to determine whether loss of cell proliferation and survival that had been observed with ACC inhibition was a direct result of FA synthesis inhibition and lipid depletion, our laboratory recently analyzed the role of ACC in NSCLC cells using CRISPR-Cas9 (Svensson et al. 2016). Consistent with the lipogenic phenotype that has been observed in tumors, we found that ACC1 expression levels were high in the panel of NSCLC cells that we analyzed and interestingly were significantly higher than ACC2, which had expression ranging from low to undetectable. CRISPR-Cas9 deletion of ACC1 led to pronounced proliferation defects and cell death, which was completely rescued by the exogenous addition of the fatty acid palmitate to the media, suggesting that the FA synthesis was impaired by ACC1 deletion. Metabolic tracing experiments to quantitate fatty acid production revealed that ACC1 deletion led to a complete loss of de novo FA synthesis, consistent with the critical role of ACC1 in lipogenesis. When we implanted ACC1-deleted NSCLC cells subcutaneously into mice, we found that tumor growth was severely impaired relative to the growth of cells bearing endogenous ACC1 expression. However, tumor growth in ACC1-deleted cells was completely rescued by stable re-expression of ACC1 into deleted cells. Taken together, our findings revealed that ACC1 mediates a metabolic liability of NSCLC, providing the rationale for targeted pharmacologic inhibition of ACC.

Consistent with the important role of fatty acid biosynthesis in cancer and consistent with our RNA-sequencing profiling, the expression of ACC1 has been shown to be frequently up-regulated in several tumor types (Witters et al. 1994; Milgraum et al. 1997; Swinnen et al. 2000; Yahagi et al. 2005). Conversely, and consistent with our studies describing low expression levels of ACC2 mRNA in NSCLC cells, the expression or activation of ACC2 has been found to be down-regulated in tumors (Corbet et al. 2016; German et al. 2016). This may be due to the important role of FAOxn in tumors to support tumor growth and survival (Carracedo et al. 2013). ACC2 inactivation in tumors can occur via phosphorylation by AMPK and recent evidence suggests that ACC2 activity is also modulated by hydroxylation (German et al. 2016). Hydroxylation of ACC2 by prolyl hydroxylase 3 (PHD3) was required for ACC2 activation to repress FAOxn and cancer cell proliferation, and it was found that cancer cells down-regulate expression of PHD3, which directly correlated with loss of ACC2 activity. Additionally, ACC2 expression may be regulated by the acetylome, providing an epigenetic mechanism for ACC2 downregulation in tumors (Corbet et al. 2016). Ultimately, despite the fact that $\mathrm{ACC} 1$ and $\mathrm{ACC} 2$ function can be redundant, certain conditions may still allow for concomitant activation of FA synthesis and FAOxn to support tumor growth.

FAOxn inhibition itself is a focus of cancer therapy and inhibition of FAOxn has shown therapeutic efficacy in cancer models (Samudio et al. 2010; Camarda et al. 2016). This may present a conundrum when discussing the value of ACC inhibition as a strategy for cancer therapy as an ACC2 inhibitor would be expected to promote FAOxn, which in theory may enhance tumor growth rather than inhibit it. However, this conundrum can be solved by development of compounds that allow the simultaneous inhibition of ACC1 and ACC2. In principle, a dual ACC1/-2 inhibitor would result in FA synthesis inhibition and induction of FAOxn, which should result in severe lipid depletion in tumors by shutting off fatty acid production and enhancing fatty acid degradation. Thus, if tumors require the mass abundance of lipids to support cell growth, then the induction of FAOxn that may support tumor growth would be negated by the severe lipid depletion achieved by ACC 1 and ACC2 inhibition, which ultimately should repress tumor growth.

\section{ACC: OLD TARGET, NEW DRUG}

Because of the pivotal role of ACC in regulating fatty acid metabolism, ACC has been under intense investigation as a clinical drug target in several metabolic diseases, including nonalcoholic fatty liver disease (NAFLD) and nonalcoholic steatohepatitis (NASH), and, correspondingly, ACC has been the focus of several clinical pipelines in the pharmaceutical industry. Inhibition of FA synthesis and stimulation of FAOxn via ACC1/-2 inhibition may favorably affect the morbidity and mortality associated with metabolic syndromes (Wakil and AbuElheiga 2009). To this end, several isoform nonselective ACC inhibitors have been described to date. However, because of the high degree of hydrophobicity in the regions of ACC that these inhibitors interact, they lack the optimal drug-like properties that are required for their clinical use. Recently, however, the development of small-molecule ACC inhibitors with more favorable drug-like properties was described by Harriman et al. (2016). In an effort to identify potent inhibitors of ACC1/-2 that display enhanced pharmacokinetics (PK) and pharmacodynamics (PD), Harriman et al. targeted the dimerization site in the $\mathrm{BC}$ domain of the enzyme in an attempt to identify novel allosteric protein-protein interaction inhibitors of ACC. Using state-of-the-art structurebased drug design, Harriman et al. identified a series of allosteric ACC1/-2 dimerization inhibitors and reported 
the ability of the liver selective compound ND-630 to reduce hepatic steatosis, improve insulin sensitivity, and reduce weight gain in experiment animal models of metabolic disease. Our own laboratory recently described the ability of another member of this series, ND-646, a more broadly distributed ACC inhibitor, to repress FA synthesis and tumor growth of NSCLC in genetically engineered preclinical models (Svensson et al. 2016).

\section{MECHANISM OF ACTION OF ND-646}

ND-646 and other members of the ACC inhibitor series developed by Harriman et al. function as allosteric inhibitors of ACC1 and ACC2, by preventing the dimerization that is required for activity (Harriman et al. 2016; Svensson et al. 2016). The site that ND-646 binds to in the BC domain of ACC is of importance, as it is the exact same site that the AMPK phosphorylated serine of ACC interacts to inhibit dimerization and activity of ACC. Thus, ACC inhibition by ND-646 mimics physiological inhibition of ACC by AMPK. Phosphorylation of conserved serine residues by AMPK in ACC1 (mouse serine 79, human serine 117) and ACC2 (mouse serine 212, human serine 222) in the A-domain tail of the BC domain promotes a conformational change of the tail and binding of the phospho-peptide to the BC dimer interface (Fig. 2). Binding of the phosphorylated serine to the $\mathrm{BC}$ domain disrupts dimerization of ACC and promotes the formation of the inactive monomer. Given that ND-646 binds to the same site in the BC domain of ACC to analogously disrupt enzyme dimerization, it also fortuitously facilitates analysis of the phosphorylation state of ACC as a biomarker to determine whether ND-646 is bound. When ND-646 binds to the BC domain, it prevents binding of the phosphorylated serine to the same site, which results in constitutive dephosphorylation of ACC as the phosphate is no longer protected from protein phosphatases (Fig. 2). Thus, phosphorylated ACC is not detected when ND-646 is bound and can be used as an efficient biomarker in cells and tissues (Svensson et al. 2016).

\section{ACC REGULATION OF FATTY ACID SYNTHESIS AS A METABOLIC LIABILITY OF NSCLC}

The development of ND-646 as a broadly distributed ACC inhibitor with good drug-like properties enabled us to characterize the in vivo PD properties of ND-646 and to determine whether ACC inhibition in tumors in vivo would be therapeutically beneficial. To determine whether ACC inhibition by ND-646 would lead to decreased de novo fatty acid synthesis in autochthonous tumors in vivo, our laboratory, in collaboration with the laboratory of Christian Metallo, performed metabolic labeling analyses in genetically engineered mouse models of NSCLC using deuterated water $\left({ }^{2} \mathrm{H}_{2} \mathrm{O}\right) .{ }^{2} \mathrm{H}_{2} \mathrm{O}$ is incorporated into a number of biosynthetic intermediates that are required for fatty acid production and only labels fatty acids that are newly synthesized (Lee et al. 2000). Thus, quantitation of deuterium-labeled fatty acids in harvested tumors provides a powerful method to measure the synthesis rates of newly made fatty acids. The ability to administer deuterated water directly into the drinking water of mice may offer advantages over alternative approaches to quantitate metabolic flux in vivo, as it is a facile procedure and well tolerated. Consistent with the studies of Medes et al. more than 60 years ago, we found that lung tumors from $\mathrm{Kras}^{\mathrm{G} 12 \mathrm{D} /+}$; Trp53 $3^{-/-}$(KP), and Kras ${ }^{\mathrm{G} 12 \mathrm{D}}$; LKB1 $^{-/-}$ (KL) mouse models were undergoing high rates of de novo fatty acid synthesis (Svensson et al. 2016). In our unpublished studies, we also directly compared the synthesis rates in tumors of KP mice to livers in the same animals and found that the rate of fatty acid synthesis of the most predominant saturated fatty acid palmitate was higher in the tumor tissue, supporting the original conclusions of Medes et al. and suggesting that this high rate of synthesis may be required to support the fast growth of these tumors. We found that treatment of lung tumors with ND-646 led to a significant decrease in the rate of fatty acid synthesis. Furthermore, plasma levels of free fatty acids were also significantly reduced in ND-646-treated mice, demonstrating the ability of ND-646 to inhibit FA synthesis in other tissues. To the best of our knowledge, our study describing these effects is the first published report of an ACC inhibitor with these pharmacodynamic properties in vivo.

Given that ND-646 was able to significantly inhibit fatty acid synthesis in lung tumors in these autochthonous models, it led us to investigate whether this inhibition would affect the growth of the tumors after chronic dosing. We set up large-scale preclinical trials in KP and KL mice to test the therapeutic efficacy of ND-646, as a single agent and also in combination with the standard of care chemotherapeutic agent carboplatin. We recently reported the results from these trials, where we found that KP mice were particularly sensitive to ND-646 monotherapy and that the combination of ND-646 and carboplatin led to striking suppression of lung tumor growth in both KP and KL mouse models. In summary, our study showed that ACC inhibition in biologically relevant preclinical mouse models of NSCLC led to suppression of fatty acid synthesis and tumor growth, revealing that ACC mediates a metabolic liability for this particular subset of cancers (Fig. 3).

\section{CONCLUSION AND FUTURE PERSPECTIVES}

Medes et al. concluded more than 60 years ago that the elevated rates of de novo lipogenesis they measured in tumors was probably required to support the rapid proliferation of cancer cells. Our work further supports this model and demonstrates the therapeutic viability of targeting this pathway (Svensson et al. 2016).

An open question that remains from our study is understanding whether decreased levels of plasma free fatty acids that is caused by ND-646 treatment contributes to the antitumor efficacy evoked by ACC inhibition (Fig. 3). We found that chronic 6 weeks of dosing of ND-646 led to significant reductions in the levels of plasma free fatty 

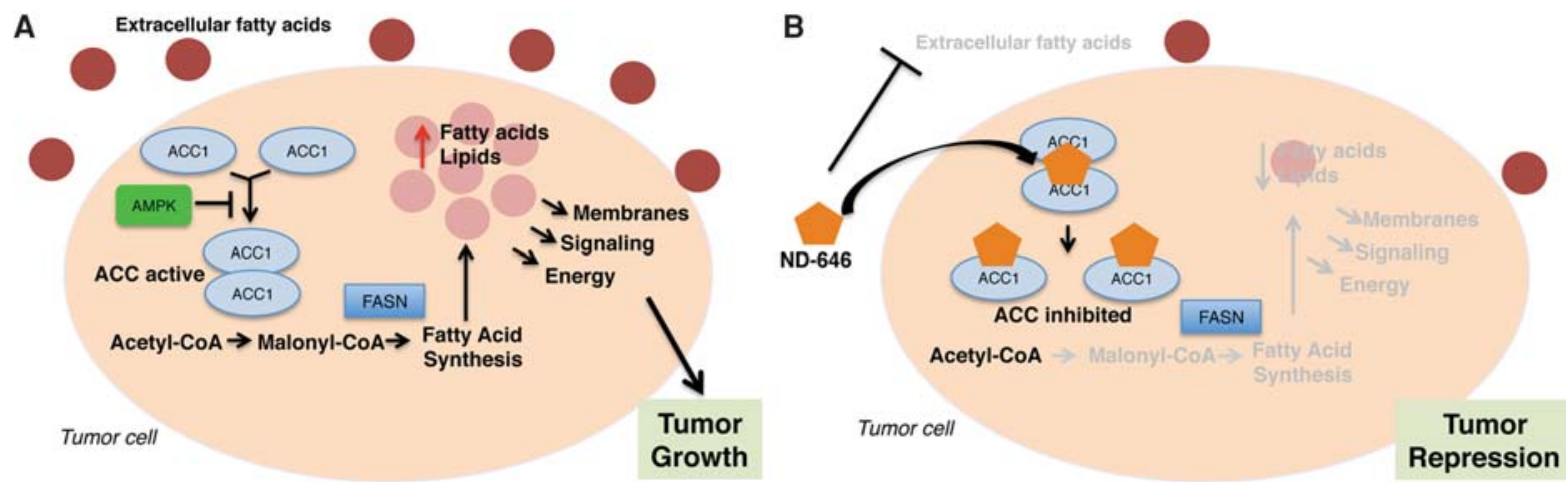

Figure 3. Model depicting the therapeutic mechanism of action of ND-646 in non-small cell lung cancer. $(A)$ Lung tumors with active acetyl-CoA carboxylase (ACC) undergo high rates of de novo FA synthesis to provide fatty acids and lipids to support tumor growth. $(B)$ ND-646 treatment inhibits ACC activity and suppresses fatty acid synthesis and tumor growth and also lowers plasma levels of fatty acids. AMPK, AMP-activated protein kinase; FASN, fatty acid synthase.

acids and given the ability of exogenous palmitate to rescue the effects of ACC inhibition in vitro suggests that the levels of fatty acids that are taken up by autochthonous tumors are limiting and unable to compensate for the loss of de novo synthesis. This leads to the possibility that the antitumor effects of ND-646 occur by at least a two-prong mechanism: (1) direct inhibition of FA synthesis in tumors, and (2) inhibition of FA synthesis in the tumor microenvironment and other tissues such as the liver, which is a major contributor to plasma fatty acid levels, thus reducing the ability of circulatory and microenvironmental fatty acids to rescue the effects of ND-646. The availability of the liver-directed ACC inhibitor, ND630 , may aid in determining whether the reduction in plasma free fatty acids levels alone has any effect on in vivo lung tumor growth. Additionally, whether diet modulates fatty acid bioavailability to tumors and the response to ND-646 will need to be determined.

Our study warrants further investigation of the utility of ACC inhibitors in oncology. Identification of specific tumor types and subsets of cancers that respond to ND646 will be of great interest. Furthermore, whether the mutational status of the tumors dictates the therapeutic response to ACC inhibitors will need to be examined. Our work showed that LKB1 deletion in $\mathrm{Kras}^{\mathrm{G} 12 \mathrm{D}}$ lung tumors led to increased rates of de novo fatty acid synthesis compared with p53 deletion, consistent with hyperactivation of ACC activity due to LKB1 loss; however, KL tumors were less sensitive to ND-646 as a single agent. This may be related to the absolute levels of fatty acids in the tumors and the degree of suppression by ND-646, which may dictate the therapeutic efficacy of ACC inhibition. However, KL lung tumors were exquisitely sensitized to the combined effects of ND-646 and carboplatin. Because of the genetic integrity of $p 53$ in the KL tumors, we found that carboplatin induced nuclear p53 accumulation in these tumors, which might explain the striking synergy that we observed with this combination treatment, as p53 activation is known to promote FAOxn via its induction of mRNAs for Cpt1c, Lipin1, and Acad11 (Assaily et al. 2011; Zaugg et al. 2011; Jiang et al. 2015).
Thus, the ability of ND-646 to inhibit FA synthesis, combined with carboplatin-induced p53-dependent FAOxn, would be expected to significantly deplete the cellular pools of fatty acids that are needed for cellular division. Given that ND-646 inhibits both ACC1 and ACC2, ND646 by itself would be expected to inhibit FA synthesis and promote FAOxn; however, the majority of tumor cell lines that we have analyzed express little to no endogenous ACC2.

Although not directly assessed in our study with ND646, ACC inhibition with ND-630 led to potent suppression of malonyl-CoA production (Harriman et al. 2016). However, whether the intracellular levels of acetyl-CoA are affected by decreased conversion into malonyl-CoA has not been analyzed. Although we showed that non-FA synthesis pathways that use acetyl-CoA, such as cholesterol synthesis, were unaffected, we did not determine whether acetylation of histones were affected, as has been described with genetic deletion of ACC in yeast and mouse liver (Galdieri and Vancura 2012; Chow et al. 2014). It will be of great interest to determine these effects and whether ACC provides an additional link between fatty acid metabolism and epigenetic control of transcriptional regulation.

Finally, given that many oncogenic proteins are modulated by lipidation, it raises the intriguing possibility that the antitumor activity of ND-646 might also be explained in part by decreased posttranslational modification of Kras, which is the oncogenic driver of NSCLC growth in our models. Kras undergoes extensive posttranslational modification, including farnesylation and palmitoylation (Ahearn et al. 2012); thus, whether loss of FA synthesis through ACC inhibition affects these modifications and the signaling ability of Kras should be investigated.

In conclusion, the development of ND-646 as a potent and specific ACC inhibitor has revealed that ACC mediates a metabolic liability of cancer. The continued discovery of compounds targeting other members of the FA synthesis pathway and the elucidation of new targets will shed light on whether inhibition of FA synthesis will translate from bench to bedside in oncology. 


\section{ACKNOWLEDGMENTS}

R.J.S. holds the William R. Brody Chair and is a professor in the Molecular and Cell Biology Department at the Salk Institute of Biological Studies. The work from our laboratory described in this review is supported by grants from the National Institutes of Health (R01CA172229, P01CA120964), the Samuel Waxman Cancer Research Foundation, and The Leona M. and Harry B. Helmsley Charitable Trust (grant \#2012-PGMED002). R.U.S. was supported by a postdoctoral fellowship from the American Cancer Society (grant ACS\#124183-PF-13-023-01-CSM).

\section{REFERENCES}

Abramson HN. 2011. The lipogenesis pathway as a cancer target. J Med Chem 54: 5615-5638.

Ahearn IM, Haigis K, Bar-Sagi D, Philips MR. 2012. Regulating the regulator: Post-translational modification of RAS. Nat Rev Mol Cell Biol 13: 39-51.

Alli PM, Pinn ML, Jaffee EM, McFadden JM, Kuhajda FP. 2005. Fatty acid synthase inhibitors are chemopreventive for mammary cancer in neu-N transgenic mice. Oncogene 24: $39-46$.

Alwarawrah Y, Hughes P, Loiselle D, Carlson DA, Darr DB, Jordan JL, Xiong J, Hunter LM, Dubois LG, Thompson JW, et al. 2016. Fasnall, a selective FASN inhibitor, shows potent anti-tumor activity in the MMTV-Neu model of HER2 ${ }^{+}$ breast cancer. Cell Chem Biol 23: 678-688.

Assaily W, Rubinger DA, Wheaton K, Lin Y, Ma W, Xuan W, Brown-Endres L, Tsuchihara K, Mak TW, Benchimol S. 2011. ROS-mediated p53 induction of Lpin1 regulates fatty acid oxidation in response to nutritional stress. Mol Cell 44: 491-501.

Bauer DE, Hatzivassiliou G, Zhao F, Andreadis C, Thompson CB. 2005. ATP citrate lyase is an important component of cell growth and transformation. Oncogene 24: 6314-6322.

Beckers A, Organe S, Timmermans L, Scheys K, Peeters A, Brusselmans K, Verhoeven G, Swinnen JV. 2007. Chemical inhibition of acetyl-CoA carboxylase induces growth arrest and cytotoxicity selectively in cancer cells. Cancer Res 67: 8180-8187.

Benjamin DI, Li DS, Lowe W, Heuer T, Kemble G, Nomura DK. 2015. Diacylglycerol metabolism and signaling is a driving force underlying FASN inhibitor sensitivity in cancer cells. ACS Chem Biol 10: 1616-1623.

Bianchi A, Evans JL, Iverson AJ, Nordlund AC, Watts TD, Witters LA. 1990. Identification of an isozymic form of acetyl-CoA carboxylase. J Biol Chem 265: 1502-1509.

Brusselmans K, De Schrijver E, Verhoeven G, Swinnen JV. 2005. RNA interference-mediated silencing of the acetylCoA-carboxylase- $\alpha$ gene induces growth inhibition and apoptosis of prostate cancer cells. Cancer Res 65: 6719-6725.

Camarda R, Zhou AY, Kohnz RA, Balakrishnan S, Mahieu C, Anderton B, Eyob H, Kajimura S, Tward A, Krings G, et al 2016. Inhibition of fatty acid oxidation as a therapy for MYCoverexpressing triple-negative breast cancer. Nat Med 22: 427-432.

Carling D, Clarke PR, Zammit VA, Hardie DG. 1989. Purification and characterization of the AMP-activated protein kinase. Copurification of acetyl-CoA carboxylase kinase and 3-hydroxy-3-methylglutaryl-CoA reductase kinase activities. Eur J Biochem 186: 129-136.

Carracedo A, Cantley LC, Pandolfi PP. 2013. Cancer metabolism: Fatty acid oxidation in the limelight. Nat Rev Cancer 13: 227-232.

Chajes V, Cambot M, Moreau K, Lenoir GM, Joulin V. 2006. Acetyl-CoA carboxylase $\alpha$ is essential to breast cancer cell survival. Cancer Res 66: 5287-5294.
Chen HW, Chang YF, Chuang HY, Tai WT, Hwang JJ. 2012. Targeted therapy with fatty acid synthase inhibitors in a human prostate carcinoma LNCaP/tk-luc-bearing animal model. Prostate Cancer Prostatic Dis 15: 260-264.

Cho YS, Lee JI, Shin D, Kim HT, Cheon YH, Seo CI, Kim YE, Hyun YL, Lee YS, Sugiyama K, et al. 2008. Crystal structure of the biotin carboxylase domain of human acetyl-CoA carboxylase 2. Proteins 70: 268-272.

Chow JDY, Lawrence RT, Healy ME, Dominy JE, Liao JA, Breen DS, Byrne FL, Kenwood BM, Lackner C, Okutsu S, et al. 2014. Genetic inhibition of hepatic acetyl-CoA carboxylase activity increases liver fat and alters global protein acetylation. Mol Metab 3: 419-431.

Comerford SA, Huang Z, Du X, Wang Y, Cai L, Witkiewicz AK, Walters H, Tantawy MN, Fu A, Manning HC, et al. 2014. Acetate dependence of tumors. Cell 159: 1591-1602.

Corbet C, Pinto A, Martherus R, Santiago de Jesus JP, Polet F, Feron O. 2016. Acidosis drives the reprogramming of fatty acid metabolism in cancer cells through changes in mitochondrial and histone acetylation. Cell Metab 24: 311-323.

Corominas-Faja B, Cuyas E, Gumuzio J, Bosch-Barrera J, Leis O, Martin AG, Menendez JA. 2014. Chemical inhibition of acetyl-CoA carboxylase suppresses self-renewal growth of cancer stem cells. Oncotarget 5: 8306-8316.

Duvel K, Yecies JL, Menon S, Raman P, Lipovsky AI, Souza AL, Triantafellow E, Ma QC, Gorski R, Cleaver S, et al. 2010. Activation of a metabolic gene regulatory network downstream of mTOR complex 1. Mol Cell 39: 171-183.

Fullerton MD, Galic S, Marcinko K, Sikkema S, Pulinilkunnil T, Chen ZP, O'Neill HM, Ford RJ, Palanivel R, O'Brien M, et al. 2013. Single phosphorylation sites in Acc1 and Acc2 regulate lipid homeostasis and the insulin-sensitizing effects of metformin. Nat Med 19: 1649-1654.

Galdieri L, Vancura A. 2012. Acetyl-CoA carboxylase regulates global histone acetylation. J Biol Chem 287: 2386523876.

German NJ, Yoon H, Yusuf RZ, Murphy JP, Finley LWS, Laurent G, Haas W, Satterstrom FK, Guarnerio J, Zaganjor E, et al. 2016. PHD3 loss in cancer enables metabolic reliance on fatty acid oxidation via deactivation of ACC2. Mol Cell 63: 1006-1020.

Griffiths B, Lewis CA, Bensaad K, Ros S, Zhang Q, Ferber EC, Konisti S, Peck B, Miess H, East P, et al. 2013. Sterol regulatory element binding protein-dependent regulation of lipid synthesis supports cell survival and tumor growth. Cancer Metab 1: 3 .

Hanai J, Doro N, Sasaki AT, Kobayashi S, Cantley LC, Seth P, Sukhatme VP. 2012. Inhibition of lung cancer growth: ATP citrate lyase knockdown and statin treatment leads to dual blockade of mitogen-activated protein kinase (MAPK) and phosphatidylinositol-3-kinase (PI3K)/AKT pathways. J Cell Physiol 227: 1709-1720.

Harada N, Oda Z, Hara Y, Fujinami K, Okawa M, Ohbuchi K, Yonemoto M, Ikeda Y, Ohwaki K, Aragane K, et al. 2007. Hepatic de novo lipogenesis is present in liver-specific ACC1deficient mice. Mol Cell Biol 27: 1881-1888.

Harriman G, Greenwood J, Bhat S, Huang X, Wang R, Paul D, Tong L, Saha AK, Westlin WF, Kapeller R, et al. 2016. Acetyl-CoA carboxylase inhibition by ND-630 reduces hepatic steatosis, improves insulin sensitivity, and modulates dyslipidemia in rats. Proc Natl Acad Sci 113: E1796-E1805.

Hatzivassiliou G, Zhao FP, Bauer DE, Andreadis C, Shaw AN, Dhanak D, Hingorani SR, Tuveson DA, Thompson CB. 2005. ATP citrate lyase inhibition can suppress tumor cell growth. Cancer Cell 8: $311-321$.

Horton JD. 2002. Sterol regulatory element-binding proteins: Transcriptional activators of lipid synthesis. Biochem Soc Trans 30: $1091-1095$.

Jiang D, LaGory EL, Kenzelmann Broz D, Bieging KT, Brady CA, Link N, Abrams JM, Giaccia AJ, Attardi LD. 2015. Analysis of p53 transactivation domain mutants reveals Acad11 as a metabolic target important for p53 pro-survival function. Cell Rep 10: 1096-1109. 
Kim KH. 1997. Regulation of mammalian acetyl-coenzyme A carboxylase. Annu Rev Nutr 17: 77-99.

Kim J, DeBerardinis RJ. 2016. Blocking fatty acid synthesis reduces lung tumor growth in mice. Nat Med 22: 10771078.

Kuhajda FP, Jenner K, Wood FD, Hennigar RA, Jacobs LB, Dick JD, Pasternack GR. 1994. Fatty acid synthesis: A potential selective target for antineoplastic therapy. Proc Natl Acad Sci 91: 6379-6383.

Lee WN, Bassilian S, Lim S, Boros LG. 2000. Loss of regulation of lipogenesis in the Zucker diabetic (ZDF) rat. Am J Physiol Endocrinol Metab 279: E425-E432.

Lee JV, Carrer A, Shah S, Snyder NW, Wei S, Venneti S, Worth AJ, Yuan Z-F, Lim H-W, Liu S, et al. 2014. Akt-dependent metabolic reprogramming regulates tumor cell histone acetylation. Cell Metab 20: 306-319.

Li Y, Xu S, Mihaylova MM, Zheng B, Hou X, Jiang B, Park O, Luo Z, Lefai E, Shyy JY, et al. 2011. AMPK phosphorylates and inhibits SREBP activity to attenuate hepatic steatosis and atherosclerosis in diet-induced insulin-resistant mice. Cell Metab 13: 376-388.

Loftus TM, Jaworsky DE, Frehywot GL, Townsend CA, Ronnett GV, Lane MD, Kuhajda FP. 2000. Reduced food intake and body weight in mice treated with fatty acid synthase inhibitors. Science 288: 2379-2381.

Luo J, Hong Y, Lu Y, Qiu S, Chaganty BK, Zhang L, Wang X, Li Q, Fan Z. 2016. Acetyl-CoA carboxylase rewires cancer metabolism to allow cancer cells to survive inhibition of the Warburg effect by cetuximab. Cancer Lett 384: 39-49.

Lyssiotis CA, Cantley LC. 2014. Acetate fuels the cancer engine. Cell 159: 1492-1494.

Mashimo T, Pichumani K, Vemireddy V, Hatanpaa KJ, Singh DK, Sirasanagandla S, Nannepaga S, Piccirillo SG, Kovacs Z, Foong C, et al. 2014. Acetate is a bioenergetic substrate for human glioblastoma and brain metastases. Cell 159: $1603-$ 1614.

McGarry JD, Leatherman GF, Foster DW. 1978. Carnitine palmitoyltransferase. I. The site of inhibition of hepatic fatty acid oxidation by malonyl-CoA. J Biol Chem 253: 41284136.

Medes G, Thomas A, Weinhouse S. 1953. Metabolism of neoplastic tissue. IV. A study of lipid synthesis in neoplastic tissue slices in vitro. Cancer Res 13: 27-29.

Menendez JA, Lupu R. 2007. Fatty acid synthase and the lipogenic phenotype in cancer pathogenesis. Nat Rev Cancer 7: $763-777$.

Menendez JA, Vellon L, Mehmi I, Oza BP, Ropero S, Colomer R, Lupu R. 2004. Inhibition of fatty acid synthase (FAS) suppresses HER2/neu (erbB-2) oncogene overexpression in cancer cells. Proc Natl Acad Sci 101: 1071510720.

Migita T, Narita T, Nomura K, Miyagi E, Inazuka F, Matsuura M, Ushijima M, Mashima T, Seimiya H, Satoh Y, et al. 2008. ATP citrate lyase: Activation and therapeutic implications in non-small cell lung cancer. Cancer Res 68: $8547-8554$.

Milgraum LZ, Witters LA, Pasternack GR, Kuhajda FP. 1997. Enzymes of the fatty acid synthesis pathway are highly expressed in in situ breast carcinoma. Clin Cancer 3: 21152120.

Pizer ES, Wood FD, Heine HS, Romantsev FE, Pasternack GR, Kuhajda FP. 1996. Inhibition of fatty acid synthesis delays disease progression in a xenograft model of ovarian cancer. Cancer Res 56: 1189-1193.

Pizer ES, Thupari J, Han WF, Pinn ML, Chrest FJ, Frehywot GL, Townsend CA, Kuhajda FP. 2000. Malonyl-coenzyme-A is a potential mediator of cytotoxicity induced by fatty-acid synthase inhibition in human breast cancer cells and xenograft. Cancer Res 60: 213-218.

Porstmann T, Santos CR, Griffiths B, Cully M, Wu M, Leevers S, Griffiths JR, Chung YL, Schulze A. 2008. SREBP activity is regulated by mTORC1 and contributes to Akt-dependent cell growth. Cell Metab 8: 224-236.
Relat J, Blancafort A, Oliveras G, Cufi S, Haro D, Marrero PF, Puig T. 2012. Different fatty acid metabolism effects of (-)epigallocatechin-3-gallate and C75 in adenocarcinoma lung cancer. BMC Cancer 12: 280.

Rohrig F, Schulze A. 2016. The multifaceted roles of fatty acid synthesis in cancer. Nat Rev Cancer 16: 732-749.

Samudio I, Harmancey R, Fiegl M, Kantarjian H, Konopleva M, Korchin B, Kaluarachchi K, Bornmann W, Duvvuri S, Taegtmeyer H, et al. 2010. Pharmacologic inhibition of fatty acid oxidation sensitizes human leukemia cells to apoptosis induction. J Clin Invest 120: 142-156.

Savage DB, Choi CS, Samuel VT, Liu ZX, Zhang D, Wang A, Zhang XM, Cline GW, Yu XX, Geisler JG, et al. 2006. Reversal of diet-induced hepatic steatosis and hepatic insulin resistance by antisense oligonucleotide inhibitors of acetylCoA carboxylases 1 and 2. J Clin Invest 116: 817-824.

Schug ZT, Peck B, Jones DT, Zhang QF, Grosskurth S, Alam IS, Goodwin LM, Smethurst E, Mason S, Blyth K, et al. 2015. Acetyl-CoA synthetase 2 promotes acetate utilization and maintains cancer cell growth under metabolic stress. Cancer Cell 27: $57-71$.

Shackelford DB, Shaw RJ. 2009. The LKB1-AMPK pathway: Metabolism and growth control in tumour suppression. Nat Rev Cancer 9: 563-575.

Shen Y, Volrath SL, Weatherly SC, Elich TD, Tong L. 2004. A mechanism for the potent inhibition of eukaryotic acetyl-coenzyme A carboxylase by soraphen A, a macrocyclic polyketide natural product. Mol Cell 16: 881-891.

Steinberg GR, Kemp BE. 2009. AMPK in health and disease. Physiol Rev 89: 1025-1078.

Svensson RU, Parker SJ, Eichner LJ, Kolar MJ, Wallace M, Brun SN, Lombardo PS, Van Nostrand JL, Hutchins A, Vera L, et al. 2016. Inhibition of acetyl-CoA carboxylase suppresses fatty acid synthesis and tumor growth of nonsmall-cell lung cancer in preclinical models. Nat Med 22: $1108-1119$.

Swinnen JV, Vanderhoydonc F, Elgamal AA, Eelen M, Vercaeren I, Joniau S, Van Poppel H, Baert L, Goossens K, Heyns W, et al. 2000. Selective activation of the fatty acid synthesis pathway in human prostate cancer. Int J Cancer 88: 176-179.

Swinnen JV, Brusselmans K, Verhoeven G. 2006. Increased lipogenesis in cancer cells: New players, novel targets. Curr Opin Clin Nutr Metab Care 9: 358-365.

Szutowicz A, Kwiatkowski J, Angielski S. 1979. Lipogenetic and glycolytic enzyme activities in carcinoma and nonmalignant diseases of the human breast. $B r J$ Cancer 39: 681-687.

Thupari JN, Pinn ML, Kuhajda FP. 2001. Fatty acid synthase inhibition in human breast cancer cells leads to malonyl-CoAinduced inhibition of fatty acid oxidation and cytotoxicity. Biochem Biophys Res Commun 285: 217-223.

Tong L, Harwood HJ Jr. 2006. Acetyl-coenzyme A carboxylases: Versatile targets for drug discovery. J Cell Biochem 99: $1476-1488$.

Ventura R, Mordec K, Waszczuk J, Wang ZT, Lai JL, Fridlib M, Buckley D, Kemble G, Heuer TS. 2015. Inhibition of de novo palmitate synthesis by fatty acid synthase induces apoptosis in tumor cells by remodeling cell membranes, inhibiting signaling pathways, and reprogramming gene expression. EBiomedicine 2: 808-824.

Wakil SJ, Abu-Elheiga LA. 2009. Fatty acid metabolism: Target for metabolic syndrome. J Lipid Res 50: S138-S143.

Wangpaichitr M, Sullivan EJ, Theodoropoulos G, Wu C, You M, Feun LG, Lampidis TJ, Kuo MT, Savaraj N. 2012. The relationship of thioredoxin-1 and cisplatin resistance: Its impact on ROS and oxidative metabolism in lung cancer cells. Mol Cancer Ther 11: 604-615.

Wellen KE, Hatzivassiliou G, Sachdeva UM, Bui TV, Cross JR, Thompson CB. 2009. ATP-citrate lyase links cellular metabolism to histone acetylation. Science 324: 1076-1080.

Witters LA, Widmer J, King AN, Fassihi K, Kuhajda F. 1994. Identification of human acetyl-CoA carboxylase isozymes in tissue and in breast cancer cells. Int J Biochem 26: 589-594. 
Yahagi N, Shimano H, Hasegawa K, Ohashi K, Matsuzaka T, Najima Y, Sekiya M, Tomita S, Okazaki H, Tamura Y, et al. 2005. Co-ordinate activation of lipogenic enzymes in hepatocellular carcinoma. Eur J Cancer 41: 1316-1322.

Yoshii Y, Furukawa T, Yoshii H, Mori T, Kiyono Y, Waki A, Kobayashi M, Tsujikawa T, Kudo T, Okazawa H, et al. 2009. Cytosolic acetyl-CoA synthetase affected tumor cell survival under hypoxia: The possible function in tumor acetyl-CoA/ acetate metabolism. Cancer Sci 100: 821-827.
Zaugg K, Yao Y, Reilly PT, Kannan K, Kiarash R, Mason J, Huang P, Sawyer SK, Fuerth B, Faubert B, et al. 2011. Carnitine palmitoyltransferase $1 \mathrm{C}$ promotes cell survival and tumor growth under conditions of metabolic stress. Genes Dev 25: $1041-1051$.

Zhao S, Torres A, Henry RA, Trefely S, Wallace M, Lee JV, Carrer A, Sengupta A, Campbell SL, Kuo YM, et al. 2016. ATP-citrate lyase controls a glucose-to-acetate metabolic switch. Cell Rep 17: 1037-1052. 


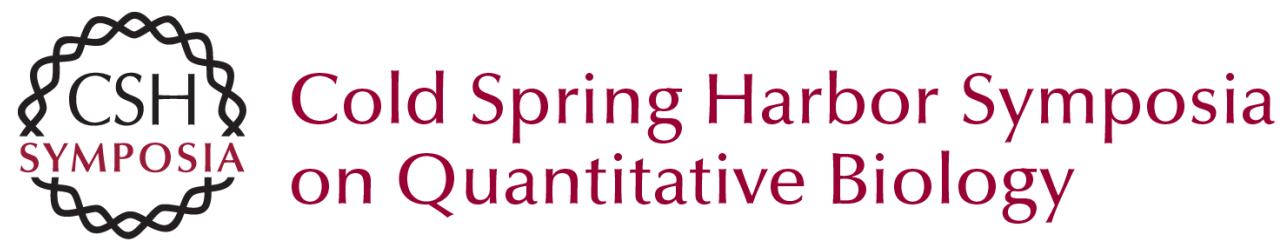

\section{Lipid Synthesis Is a Metabolic Liability of Non-Small Cell Lung Cancer}

Robert U. Svensson and Reuben J. Shaw

Cold Spring Harb Symp Quant Biol 2016 81: 93-103 originally published online January 6, 2017 Access the most recent version at doi:10.1101/sqb.2016.81.030874

References This article cites 73 articles, 20 of which can be accessed free at: http://symposium.cshlp.org/content/81/93.full.html\#ref-list-1

Creative This article is distributed under the terms of the

Commons http://creativecommons.org/licenses/by-nc/4.0/, which permits reuse and License redistribution, except for commercial purposes, provided that the original author and source are credited.

Email Alerting Receive free email alerts when new articles cite this article - sign up in Service the box at the top right corner of the article or click here. 